\title{
Multiple Primary Cardiac Malignant Peripheral Nerve Sheath Tumors in the Left Atrium: Case Report
}

\author{
Junfei Li, M.D. ', Qiansu Chen, M.D. ${ }^{1}$, Shaomei Yu, M.D. ${ }^{2}$, Siyuan Yang, M.D. ${ }^{1}$ \\ Departments of 'Cardiac Surgery and '2Ultrasound, Affiliated Hospital of Guizhou Medical University, Guiyang, China
}

\section{ARTICLE INFO}

Received October 8, 2020

Revised October 28, 2020

Accepted October 28, 2020

Corresponding author

Siyuan Yang

Tel 86-17784950043

Fax 86-851-86773651

E-mail yangsycs@gmc.edu.cn

ORCID

https://orcid.org/0000-0002-8087-7931

\begin{abstract}
Malignant peripheral nerve sheath tumors are rare sarcomas of the heart. Herein, we report the case of a 24-year-old man who complained of dyspnea, cough, and upper left back pain. He was found to have multiple primary heart tumors obstructing the right superior pulmonary vein in the left atrium, which were diagnosed as malignant peripheral nerve sheath tumors. The patient underwent successful resection of the tumors and immunohistochemistry was utilized for diagnosis.
\end{abstract}

Keywords: Neurogenic tumor, Neurofibrosarcoma, Heart neoplasms, Case report

\section{Case report}

A 24-year-old man presented to Affiliated Hospital of Guizhou Medical University with a 20-day history of dyspnea, cough, and upper left back pain. He was previously healthy and had no relevant family history. Transthoracic echocardiography showed a large tumor inside the left atrium; however, the origin and extent of the tumor could not be visualized (Fig. 1A). A subsequent thoracic-abdominal contrast-enhanced computed tomography (CT) scan revealed a large tumoral lesion (approximately $6.9 \mathrm{~cm} \times 4.0$ $\mathrm{cm} \times 3.5 \mathrm{~cm}, 45$ Hounsfield units) inside the left atrium, extending toward and totally obstructing the right superior pulmonary vein (RSPV). Two smaller tumors were also de- tected (Fig. 1B). Tumor markers were examined without positive findings.

The patient was referred for surgical treatment. Cardiopulmonary bypass was instituted and the patient was cooled to $32^{\circ} \mathrm{C}$. The interatrial wall was opened and a giant hard mass was identified (Fig. 2A). The mass was located at the roof of the left atrium, with poor mobility and extensive adhesion to the atrial septal wall. Exploring the pulmonary vein openings based on the CT images, we found that the mass intruded upon and completely blocked the RSPV. Palpation confirmed that the distal part of the RSPV was soft, and the RSPV was incised by approximately $2 \mathrm{~cm}$. The tumor in the RSPV was completely exposed. We dissected the tumor from both the left atrium and
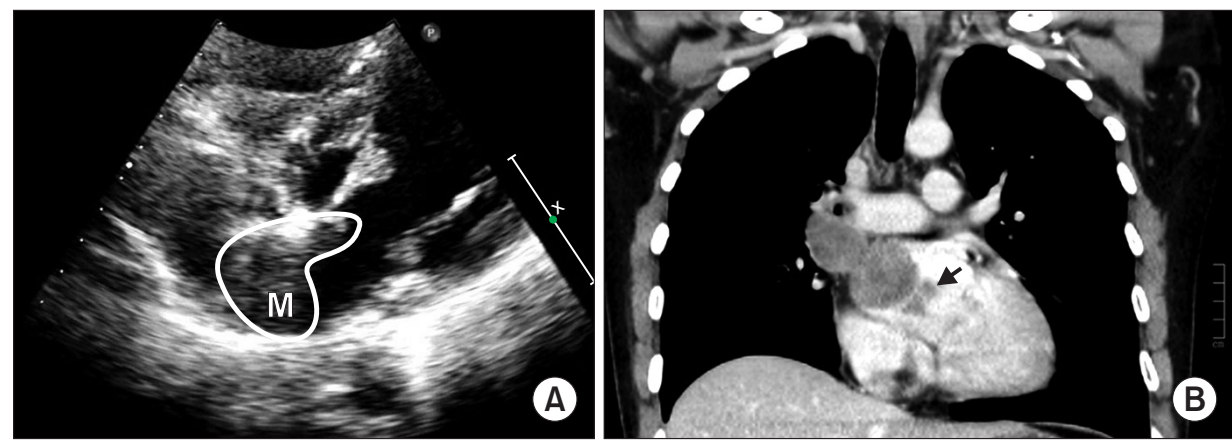

Fig. 1. (A) Transthoracic echocardiogram showed the mass (labeled as $\mathrm{M}$ ) in the left atrium. (B) Preoperative contrast-enhanced computed tomography angiography demonstrating a giant tumoral mass inside the left atrium, causing total obstruction of the right superior pulmonary vein and 2 small lesions (arrow).

Copyright $\odot$ 2021, The Korean Society for Thoracic and Cardiovascular Surgery

(c) (i) \& This is an Open Access article distributed under the terms of the Creative Commons Attribution Non-Commercial License (http://creativecommons.org/licenses/ by-nc/4.0) which permits unrestricted non-commercial use, distribution, and reproduction in any medium, provided the original work is properly cited. 

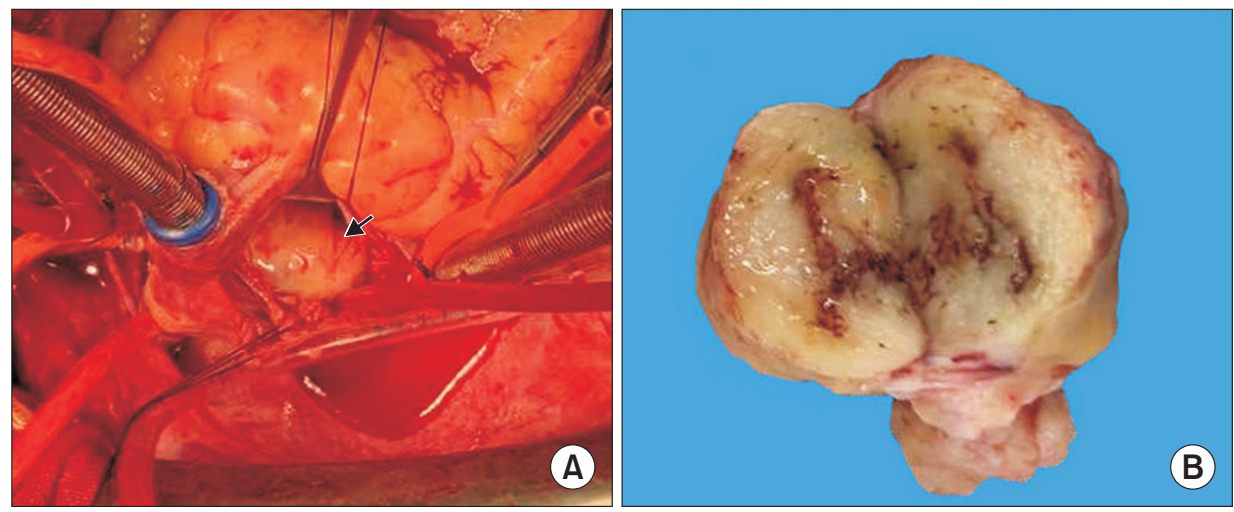

(B)
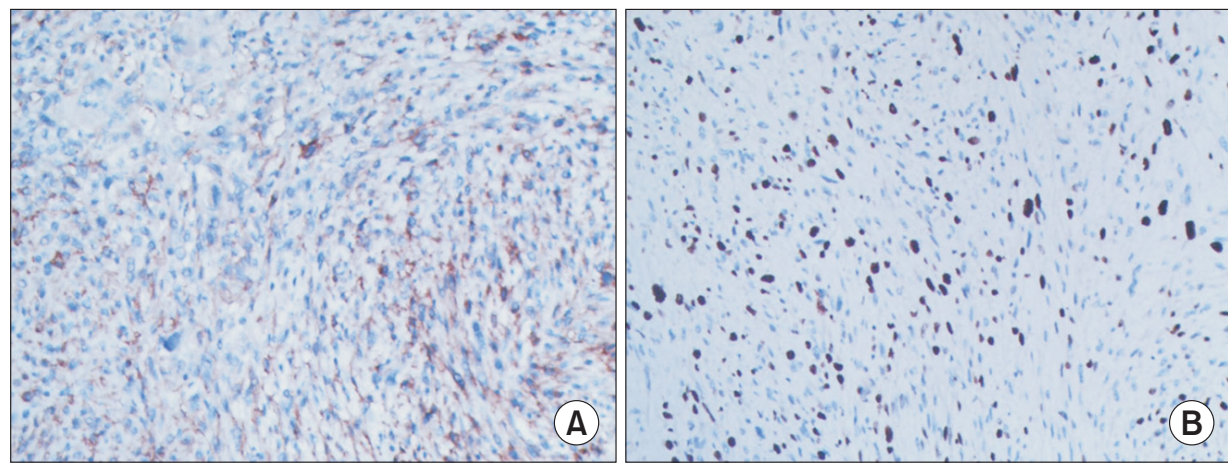

Fig. 2. (A) Intraoperative view of the tumoral mass (arrow) after opening of the interatrial wall from the right atrium. (B) The cut surface of the largest mass was glistening and myxoid, with focal hemorrhage and necrosis.

Fig. 3. Immunohistochemical staining showed positivity for vimentin $(A$, original magnification $\times 100)$ and intense expression of $\mathrm{S} 100$ (B, original magnification $\times 100$ ).
RSPV. The RSPV incision was closed with a 1-cm-wide autologous pericardial patch, and an $8-\mathrm{mm}$ probe could be passed through the RSPV. The opening of the RSPV and the left atrium were reconstructed with a pericardial patch. We detected 2 other small lesions lying on the endocardium $10 \mathrm{~mm}$ above the mitral annulus. The 2 small tumors were resected en bloc from the atrial wall. Transesophageal echocardiography after cardiopulmonary bypass showed that the RSPV was patent and the tumor had been totally removed. The biopsy of the biggest mass was glistening and myxoid, with focal hemorrhage and necrosis (Fig. 2B). The patient had an uneventful postoperative course. The pathology diagnosis was malignant peripheral nerve sheath tumor (MPNST), a rare variety of soft tissue sarcoma. The immunohistochemistry results were as follows: creatine kinase $(-)$, epithelial membrane antigen $(-)$, vimentin $(+)$ (Fig. 3A), CD34 (-), CD117 (-), DOG1 (-), desmin (-), smooth muscle actin (-), S100 (+) (Fig. 3B), Syn (-), CgA (-), CD56 $(+)$, myogenin $(-)$, MyoD1 (-), caldesmon $(-)$, CD68 (focal + ), and Ki-67 (40\%). At 6 months after the surgical correction, the patient remained alive and showed no signs of recurrence.

The study was approved by the Affiliated Hospital of Guizhou Medical University (IRB approval no., GY2020-
3154). This case is presented with written informed consent from the patient.

\section{Discussion}

MPNST, also known as neurofibrosarcoma, is a tumor that develops from nerve tissue. MPNST can occur anywhere in the body, but most often occurs in the deep tissue of the arms, legs, or trunk [1]. The treatment of MPNST begins with surgery to remove as much of the tumor as possible. Radiation therapy and chemotherapy might be useful in some patients to decrease the chance of recurrence [2]. Approximately 25\%-50\% of MPNSTs are associated with a genetic condition known as neurofibromatosis type 1 [3]. Surgical excision is the gold standard, with median survival ranging from 3 months to 1 year due to its high recurrence rate and metastasis. MPNST is considered an aggressive tumor because there is up to a $65 \%$ chance of recurrence after surgery, and an approximately $40 \%$ chance of metastasis to distant parts of the body. Primary cardiac MPNST is very rare. In our case, the patient had multiple lesions, which is even more uncommon. Ogechuk$\mathrm{wu}$ and colleagues reported a unique case of a primary cardiac sarcoma originating from the left atrium and ob- 
structing the right pulmonary vein [4]. Our case is the first report of multiple MPNST lesions, with 1 lesion obstructing the RSPV.

The majority of MPNSTs show a fascicular spindle cell architecture histologically, as in our case. By immunohistochemistry, approximately $50 \%$ to $90 \%$ of MPNSTs are positive for $S 100$ [5]. This same feature was seen in our case. Our case also showed intense expression of vimentin, positive expression of CD56, and focal positivity for CD68.

In conclusion, this is the first reported case of a patient with multiple MPNSTs causing total obstruction of the RSPV who underwent successful surgical correction. An immunohistochemical examination was required to confirm diagnosis. Complete dissection was crucial. Chemotherapy and radiotherapy might be useful for some patients with similar conditions.

\section{Conflict of interest}

No potential conflict of interest relevant to this article was reported.

\section{Acknowledgments}

We thank Dr. Zhu Gong for technical assistance and also sincerely mourn his passing. He was a good doctor as well as a good friend.

\section{ORCID}

Junfei Li: https://orcid.org/0000-0001-7358-3372

Qiansu Chen: https://orcid.org/0000-0001-6542-3141

Shaomei Yu: https://orcid.org/0000-0002-3835-3747

Siyuan Yang: https://orcid.org/0000-0002-8087-7931

\section{References}

1. James AW, Shurell E, Singh A, Dry SM, Eilber FC. Malignant peripheral nerve sheath tumor. Surg Oncol Clin N Am 2016;25:789802.

2. Rahman M, Cook DS, Ellis G, O'keefe PA. Malignant peripheral nerve sheath tumor of the heart. Asian Cardiovasc Thorac Ann 2006; 14:425-7.

3. Kitamura M, Wada N, Nagata S, et al. Malignant peripheral nerve sheath tumor associated with neurofibromatosis type 1, with metastasis to the heart: a case report. Diagn Pathol 2010;5:2.

4. Ogechukwu CN, Christopher N, Christoph S, Etinosasere O, Jose B. The insidious cardiac tumor: a primary left atrium intimal cardiac sarcoma in a young patient. Case Rep Cardiol 2019;2019:7245676.

5. Prifti E, Baboci A, Ikonomi M. A giant cardiac malignant peripheral nerve sheath tumor presenting with total obstruction of the superior vena cava. Ann Thorac Surg 2014;97:e7-9. 